\title{
Star-forming galaxies in low-redshift clusters: comparison of integrated properties of cluster and field galaxies ${ }^{\star}$
}

\author{
C. F. Bretherton ${ }^{1,2}$, P. A. James ${ }^{1}$, C. Moss ${ }^{1 \star \star}$, and M. Whittle ${ }^{3}$ \\ ${ }^{1}$ Astrophysics Research Institute, Liverpool John Moores University, Birkenhead CH41 1LD, UK \\ e-mail: paj@astro.livjm.ac.uk \\ 2 Carter Observatory, Wellington, New Zealand \\ 3 Department of Astronomy, University of Virginia, Charlottesville, VA 22903, USA
}

Received 11 September 2009 / Accepted 20 September 2010

\begin{abstract}
Aims. We investigate the effect of the cluster environment on the star formation properties of galaxies in 8 nearby Abell clusters. Methods. Star formation properties are determined for individual galaxies using the equivalent width of $\mathrm{H} \alpha+[\mathrm{NII}]$ line emission from narrow-band imaging. Equivalent width distributions are derived for each galaxy type in each of 3 environments - cluster, supercluster (outside the cluster virial radius) and field. The effects of morphological disturbance on star formation are also investigated.

Results. We identify a population of early-type disk galaxies in the cluster population with enhanced star formation compared to their field counterparts. The enhanced cluster galaxies frequently show evidence of disturbance, and the disturbed galaxies show marginal evidence for a higher velocity dispersion, possibly indicative of an infalling population.
\end{abstract}

Key words. galaxies: clusters: general - galaxies: evolution - galaxies: interactions

\section{Introduction}

The transformation of spiral galaxies to lenticulars in the cluster environment over the last $\sim 5$ Gyr is now well established (Butcher \& Oemler 1978, 1984; Dressler et al. 1997), but the processes responsible for bringing about this change are still the subject of vigorous discussion. One set of mechanisms centres on the removal of gas from spiral galaxies in the cluster environment, whether through collisional sweeping (Spitzer \& Baade 1951; Valluri \& Jog 1990), ram-pressure stripping of disk gas (Gunn \& Gott 1972; Cowie \& Songaila 1977; Nulsen 1982; Quilis et al. 2000), or the removal of large-scale gas reservoirs in "strangulation" or "starvation" scenarios (Larson et al. 1980; Balogh et al. 2000; Bower \& Balogh 2004), with the latter postulated to result in anaemic spirals (Bekki et al. 2002). Many studies have looked at the effect of tidal interactions either in the general cluster environment (Noguchi \& Ishibashi 1986; Lavery \& Henry 1988; Henriksen \& Byrd 1996), or in cluster sub-units (Hernquist \& Mihos 1995; Barnes \& Hernquist 1996; Bekki 1999; Gnedin 2003). Some have concluded that tidal effects lead to galaxy-wide star formation (Byrd \& Valtonen 1990); this is one of the possible consequences of repeated highvelocity galaxy-galaxy encounters, sometimes termed "harassment" (Moore et al. 1999; Mihos 2004). Low-velocity tidal encounters, on the other hand, tend to drive gas into the central regions of galaxies leading to nuclear star formation (SF) and

\footnotetext{
* Based on observations made with the Nordic Optical Telescope, operated on the island of La Palma jointly by Denmark, Finland, Iceland, Norway, and Sweden, in the Spanish Observatorio del Roque de los Muchachos of the Instituto de Astrofísica de Canarias; and with the Jacobus Kapteyn Telescope, which was operated on the island of La Palma by the Isaac Newton Group in the Spanish Observatorio del Roque de los Muchachos of the Instituto de Astrofísica de Canarias.

$\star \star$ Deceased 12th May 2010.
}

the build-up of bulges (Kennicutt et al. 1987; Mihos et al. 1992; Iono et al. 2004). It is also possible that the bulk of the evolutionary activity took place through pre-processing in galaxy groups (Zabludoff \& Mulchaey 1998) prior to the assembly of these groups into the present-day clusters.

It should be noted that, given the range of processes likely to be operating, it is not clear a priori whether the predominant effect of the cluster environment will be to suppress (through gas removal or exhaustion) or enhance (through, e.g., tidal triggering) total SF rates in disk galaxies. Observational studies have adduced evidence supporting both possibilities, with suppressed SF being found by both Balogh et al. (1998) and Hashimoto et al. (1998), whereas others (Donas et al. 1990; Moss \& Whittle 1993; Gavazzi \& Contursi 1994; Biviano et al. 1997; Moss et al. 1998; Gavazzi et al. 1998) conclude that cluster spirals have SF activity similar to or enhanced in comparison with the field population.

A comprehensive survey of the observational work undertaken in this area is beyond the scope of this paper, but it is illustrative to consider some of the approaches that have been adopted. One route is to focus on the properties of galaxies in intermediate-redshift clusters, as exemplified by Moran et al. (2007) who studied the SF activity in clusters at redshifts of 0.5. They identify a population of disk galaxies with young stellar populations but no ongoing SF, which they take as evidence for gradual curtailment of SF through "strangulation" or similar processes. However, they also identify a density threshold for intra-cluster gas, above which a single passage of even a large galaxy can lead to gas stripping and an abrupt transformation to a gas-depleted lenticular with no star formation.

A second approach is to use the statistical properties of very large numbers of low-redshift galaxies now made available by the Sloan Digital Sky Survey (SDSS). Park et al. (2007) present a study of the colours and morphologies of $>300000$ SDSS 
galaxies within $z \sim 0.1$, which they correlate with the local number density around each of the galaxies. They find the fraction of galaxies with early morphological type to be a monotonically increasing function of this number density, and propose tidal processes as the dominant mechanism of galaxy transformation.

A third method, and the one adopted in the present study, is to look in great detail at the SF properties (rates and spatial distributions) of spiral galaxies in the nearest clusters. An excellent example of this approach is the study of the Virgo cluster undertaken by Koopmann \& Kenney (2004a,b) using spatially resolved SF mapping based on $\mathrm{H} \alpha$ narrow-band imaging. They find that many of the Virgo cluster spiral galaxies show outer truncation of their SF, in comparison with a sample of field galaxies, and some show centrally-enhanced SF. They conclude that a combination of ram-pressure stripping and tidally-induced $\mathrm{SF}$ are required to explain these observations.

The present paper is part of a study that is applying techniques similar to those of Koopmann \& Kenney (2004a,b) to eight other nearby galaxy clusters. The sample definition is presented in the first paper of this series (Thomas et al. 2008) which also describes the observations (broad- and narrow-band CCD photometry) and the data reduction process. The present paper contains an analysis of the total SF properties (rates and $\mathrm{H} \alpha$ equivalent widths) of the cluster galaxies, which are compared with a field galaxy sample derived from the $\mathrm{H} \alpha$ Galaxy Survey (James et al. 2004), henceforth H $\alpha$ GS. Future papers will look at more detailed properties of SF within these galaxies, e.g. concentration indices and radial distributions.

\section{Data}

\subsection{The comparison samples}

Global parameters for all observed cluster galaxies are given in Table 2 of Thomas et al. (2008). However, for a robust comparison with the $\mathrm{H} \alpha \mathrm{GS}$ field data, the cluster data are restricted to two well defined subsamples. The first of these is a complete sample of all Sa-Sc galaxies in six of the eight survey clusters (Abell 400, 426, 569, 779, 1367, 1656) as surveyed by the Objective Prism Survey (henceforth OPS; Moss \& Whittle 2000, 2005). The second contains all emission line galaxies (ELGs) detected by the OPS in all eight clusters (i.e. the above six clusters plus Abell 262 and 347) which excludes some of the galaxies with emission lines of lower equivalent width (Thomas et al. 2008).

A full discussion of the completeness of the ELG sample as a function of $\mathrm{H} \alpha$ flux, equivalent width $(E W)$ and surface brightness is given in Thomas et al. (2008). To summarise, all three factors affect the detectability of galaxies by the OPS, with surface brightness being the most important. The ELG sample becomes significantly incomplete below an $E W$ of $2 \mathrm{~nm}$, and below an $\mathrm{H} \alpha$ flux of $3.2 \times 10^{-17} \mathrm{~W} \mathrm{~m}^{-2}$. However, the cleanest detectability threshold is given by $\mathrm{H} \alpha$ surface brightness with a limit of $4 \times 10^{-20} \mathrm{~W} \mathrm{~m}^{-2} \operatorname{arcsec}^{-2}$.

An essential requirement for this project is consistent morphological classifications across the different samples used. For many of the cluster galaxies, no literature classifications were available, and these were provided by one of the authors (MW) working from plate material as explained in Moss \& Whittle (2000). These classifications were done on the revised de Vaucouleurs system (de Vaucouleurs 1959, 1974), and intercomparisons were performed where possible, with galaxies with classifications given in the UGC (Nilson 1973) and RC3 (de Vaucouleurs et al. 1991) catalogues. These comparisons revealed no systematic offsets and a scatter of about $1 T$-type in classification, similar to the scatter found from blind repeats of the same galaxies. For the field sample, classifications were again on the de Vaucouleurs system, taken directly from the RC3 or UGC.

Moss (2006) suggests that the late type (Sa and later) cluster galaxy population has an infalling component with higher velocity dispersion than the earlier types, as well as an asymmetric velocity dispersion relative to the cluster mean. On the other hand, the early type (E-S0/a) galaxies are consistent with a virially relaxed population with a Gaussian velocity distribution. Moss (2006) therefore determines revised cluster mean velocities and velocity dispersions from only the early type objects, using biweight estimators of scale and central location. Assuming that the distribution of galaxies follows the mass distribution of the cluster and that the system is spherically symmetric, and following Lewis et al. (2002), Moss estimates virial radii for all clusters using:

$r_{\mathrm{vir}} \simeq 3.5 \sigma(1+z)^{-1.5}$,

where $r_{\text {vir }}$ is in units of $\mathrm{Mpc}$ for $\sigma$ in units of $1000 \mathrm{~km} \mathrm{~s}^{-1}$. This gives values $40 \%$ larger than the $R_{200}$ cluster radius often used as a proxy for the virial radius (e.g. by Finn et al. 2005) for standard cosmological parameters $\left(H_{0}=70 \mathrm{~km} \mathrm{~s}^{-1} \mathrm{Mpc}^{-1}, \Omega_{\Lambda}=0.7\right.$, $\Omega_{0}=0.3$ ); both definitions give a direct proportionality between virial radius and cluster velocity dispersion.

Table 1 of Moss (2006) shows revised values of cluster mean velocity, $\bar{v}$ and velocity dispersion, $\sigma$, along with virial radius, $r_{\text {vir }}$, for each sample cluster. These were used to combine the sample galaxies, from six or eight individual clusters, into one ensemble cluster, where radial distances from the centre are normalised by the virial radius, and velocities relative to the cluster mean are normalised by the cluster's velocity dispersion.

Galaxies within the two subsamples are restricted to those with velocities within $3 \sigma$ of the revised mean cluster velocity, $\bar{v}$. The data are also split into cluster galaxies, which lie within 1 $r_{\text {vir }}$ of the cluster centre, and supercluster field galaxies lying beyond $1 r_{\text {vir }}$. The Sa-Sc sample comprises 105 galaxies of which 5 have no detectable $\mathrm{H} \alpha$ emission. The ELG sample comprises 115 objects, all of which have been detected in emission in the current CCD data. Known AGN were excluded by searching the NASA/IPAC Extragalactic Database (NED) and removing all galaxies classified as Seyfert (Sy, with any numerical subtype) or LINER. Two galaxies lying close to bright stars have also been excluded.

Galaxies in the cluster and supercluster subsamples are also classified, based only on their $R$ band images, as to whether they show signs of tidal disturbance. Objects exhibiting strong tidal features and/or obvious distortion are classified as $T$, tidally disturbed; those with less obvious warps, probable tidal tails and/or some disturbance are given a classification of $T$ :, probably disturbed; an asymmetric appearance or slight distortion of outer spiral arms leads to a classification of $T::$, possibly disturbed; and galaxies with no sign of tidal disturbance are assigned no value in this category.

The field data are taken from $\mathrm{H} \alpha \mathrm{GS}$, a study of the SF properties of 327 field galaxies of all spiral and irregular types (SOa-Im, barred and unbarred), with apparent magnitudes brighter than $m_{B}=15.5$, recession velocities less than $3000 \mathrm{~km} \mathrm{~s}^{-1}$ and diameters between 1.7 and 6.0 arcmin. The full $\mathrm{H} \alpha \mathrm{GS}$ sample includes a large number of low-luminosity galaxies, but the subsample studied here was restricted to galaxies brighter than $M_{B} \sim-18.5$. All galaxies classified as AGN in NED (26 in total in $\mathrm{H} \alpha \mathrm{GS}$ ) were excluded from the sample, as was done for the cluster sample, but it is worth noting that a 
study of the mean $\mathrm{H} \alpha$ emission profiles of the $\mathrm{H} \alpha \mathrm{GS}$ galaxies (James et al. 2009) found that central unresolved components are not common, and typically contribute less than $10 \%$ of the total emission-line flux when they are present. Thus even if some low-level AGN are included in the cluster or field samples (as will almost certainly be the case), the effect on amounts and spatial distributions of emission line flux should be small. The $\mathrm{H} \alpha \mathrm{GS}$ survey does include some Virgo cluster and group galaxies, which have also been excluded from the comparison sample. The final field comparison sample includes 65 galaxies, 50 of which are of types $\mathrm{Sa}-\mathrm{Scd}$, with 4 of type S0a and the remaining 11 being late-type spirals, Sd-Sm. Absolute $B$-band magnitudes for the field data were taken uncorrected from $\mathrm{H} \alpha \mathrm{GS}$ and have been corrected for internal and external extinction. Internal extinction corrections $A_{B}$ were calculated following the methods of de Vaucouleurs et al. (1991), including both inclination- and type-dependence as follows:

$A_{B}=\alpha(T) \log (a / b)$

where $(a / b)$ is the major-to-minor axis ratio, here evaluated at the $\mu_{R}=24$ isophote, and

$\alpha(T)=1.5-0.03(T-5)^{2}$.

For galaxies with $T<0$, no internal extinction correction was applied, and for peculiar galaxies and those without spiral subtypes, a mean spiral correction of 1.3 mag was used. Although the resulting overall shapes of the cluster and field $M_{B}$ distributions differ, they are well matched in mean and range.

Comparing the morphological type distributions of the cluster and field samples, a larger fraction of early type and fewer late type spirals are seen in the cluster environment, as expected from the morphology-density relation. The cluster ELG sample also contains three type categories not covered by the $\mathrm{H} \alpha \mathrm{GS}$ survey. Three ELGs have types E-S0 (S0-), 15 are classified as peculiar (Pec), which lie outside the Hubble sequence, and 23 are spirals of uncertain type (S...). The ELG sample also includes two galaxies with no type information, which are excluded from much of the following analysis.

$\mathrm{H} \alpha \mathrm{GS}$ observations are restricted to galaxies with major-tominor axis ratios of less than or equal to 4.0 , in order to exclude highly inclined objects $\left(i \gtrsim 81^{\circ}\right)$, where extinction effects are likely to be strongest. However, a Kendall rank test on the complete cluster Sa-Sc sample shows no significant dependence $(\tau=-0.09$, probability $=0.16)$ of $E W$ on inclination within this survey, and therefore no axis ratio cut is applied to the cluster samples.

\subsection{Observational data}

The data used are derived from CCD images taken in broad-band $R$ and narrow-band $\mathrm{H} \alpha$ filters, using the $1.0 \mathrm{~m}$ Jacobus Kapteyn Telescope (JKT) and the $2.6 \mathrm{~m}$ Nordic Optical Telescope (NOT), both situated on the island of La Palma. The instrumentation used on both the JKT and NOT and the resulting cluster galaxy photometry are described fully in Thomas et al. (2008), and the equivalent for the comparison field sample in James et al. (2004), so the details will not be repeated here. It should be noted that the line fluxes and equivalent widths used are for the $\mathrm{H} \alpha$ line and the neighbouring [NII] lines. The narrow-band imaging was continuum-subtracted using appropriately-scaled and aligned $R$-band images, which generally gives good results but inevitably gives substantial errors for galaxies with low equivalent width emission.

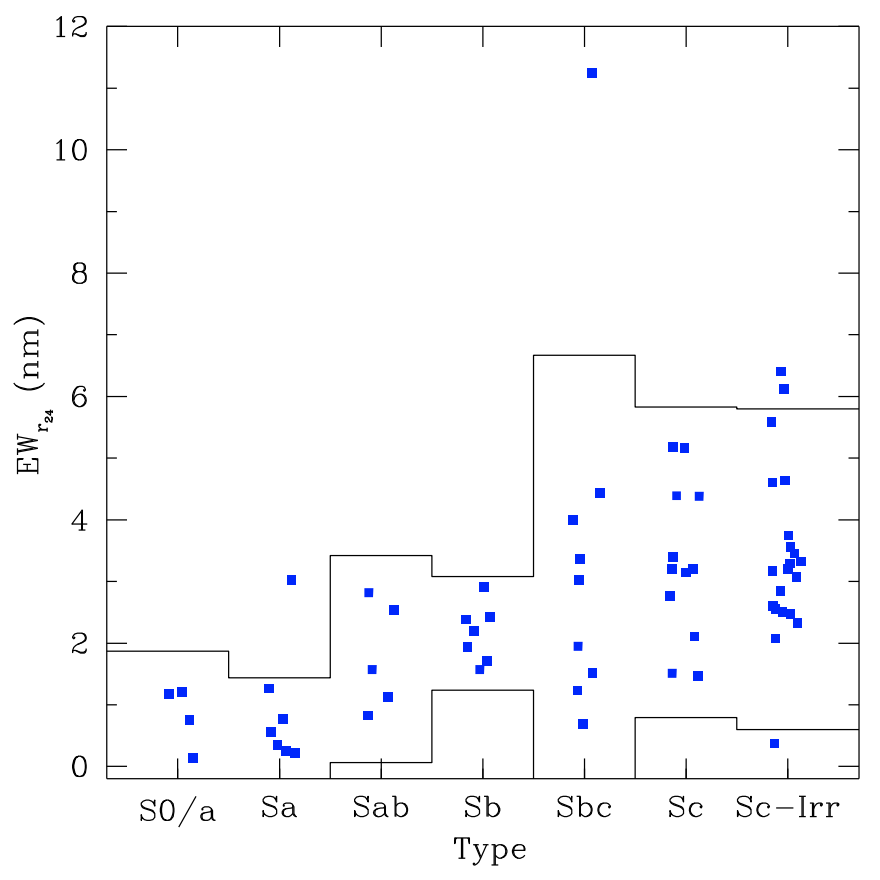

Fig. 1. Global $E W$ vs. morphological type for the full field comparison sample. Solid lines show the $2 \sigma$ limits of the field population. Typical errors in $E W$ are $10-15 \%$ for high $E W(>2 \mathrm{~nm})$ and $25-35 \%$ for low $E W(<2 \mathrm{~nm})$ galaxies (Shane \& James 2002).

\section{Distribution of equivalent widths}

Figure 1 shows the distribution of total $E W$ values with type for the comparison field sample, where the total $E W$ for each galaxy is taken as the $E W$ within the $R=24 \mathrm{mag} / \mathrm{sq}$. arcsec isophotal radius $\left(r_{24}\right)$. A biweight estimator method is used to calculate the mean $E W$ and standard deviation for each type and the $2 \sigma$ limits of the field data are plotted as solid lines in the figure.

The majority of field points lie within these limits. It is worth noting here that the field galaxy with the highest $E W$ in the field sample, and which lies well beyond the $2 \sigma$ limit, is the Sbc galaxy UGC5786 (NGC 3310). This is a well-studied example of a local UV-bright starburst with a complex peculiar morphology (Kinney et al. 1993; Conselice et al. 2000), including a "bow and arrow" structure in the outer regions (Walker \& Chincarini 1967; Balick \& Heckman 1981; Bertola \& Sharp 1984; Mulder et al. 1995) most likely caused by a recent merger with a smaller galaxy (Balick \& Heckman 1981; Mulder \& van Driel 1996; Conselice et al. 2000).

Figure 2 shows similar plots for the cluster and supercluster $\mathrm{Sa}-\mathrm{Sc}$ samples, where again the solid lines show the $2 \sigma$ limits generated from the field sample, and galaxies are split into disturbed (open points) and undisturbed (filled points) objects on each plot. It can be seen from Fig. 2a that a substantial fraction of cluster galaxies lie beyond the $2 \sigma$ field limits ( $~ 36 \%$ compared to only $5 \%$ of field $\mathrm{Sa}-\mathrm{Sc}$ galaxies). Ten cluster $\mathrm{Sa}-\mathrm{Sc}$ galaxies (13.5\% of the total) appear to have $E W$ reduced compared to the field sample. Three of these galaxies have no detected $\mathrm{H} \alpha$ emission, however, 9 of the low emission objects are $\mathrm{Sb}$ spirals for which the range in field $E W$ values is surprisingly small.

More significant are the 17 galaxies (23\%), particularly of earlier $\mathrm{Sa}-\mathrm{Sb}$ types, with $E W$ values beyond the upper field limits. This suggests that the cluster environment is causing an enhancement of star formation in some spirals. There is also some evidence that galaxies with enhanced emission may 

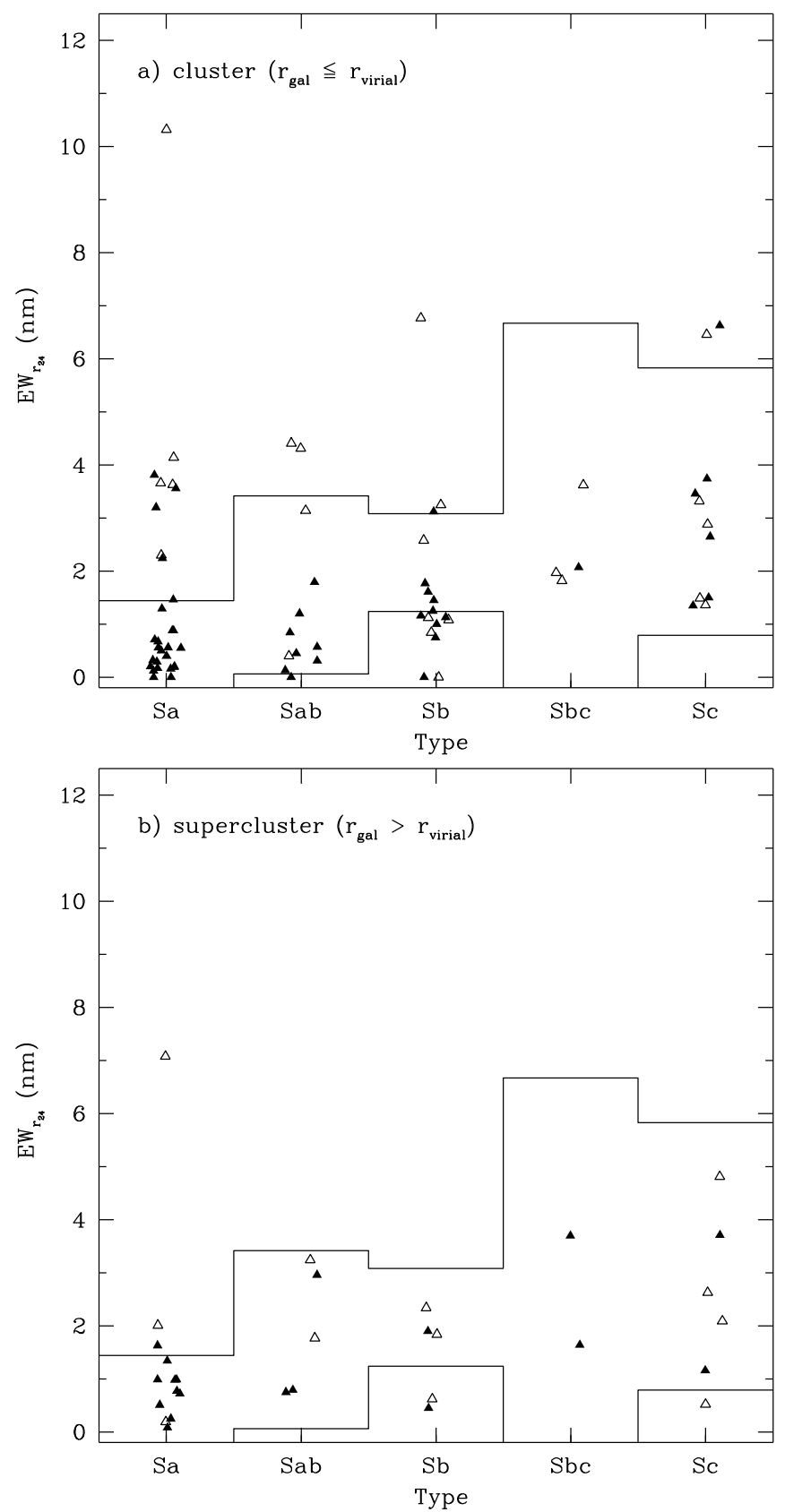

Fig. 2. Distribution of $E W$ with Hubble Type for the cluster Sa-Sc sample split into disturbed (open triangles) and undisturbed (filled triangles) objects. Solid lines show the $2 \sigma$ field limits from Fig. 1. Typical errors in $E W$ are $\sim 5-15 \%$ for high $E W(>2 \mathrm{~nm}), 15-25 \%$ for moderate $E W$ $(1-2 \mathrm{~nm})$ and $25-100 \%$ for low $E W(<1 \mathrm{~nm})$ objects.

preferentially be disturbed, with nearly $59 \%$ of enhanced galaxies showing clear signs of tidal disturbance compared to less than $23 \%$ of galaxies with no enhancement in $E W\left(\chi^{2}\right.$ probability $<0.05)$. The supercluster field sample on the other hand is similar to the true field population, with only two galaxies lying significantly above the $2 \sigma$ limits, and both of these have a disturbed appearance.

Figure 3 shows the distribution of $E W$ values with type for the cluster ELG sample, again split into cluster and supercluster field objects. Here the solid lines again show the $2 \sigma$ limits for the field sample. For the peculiar galaxies and spirals of uncertain type, the upper limit is taken as the Sbc value (shown as a dashed line) as this is the highest $2 \sigma$ value in the field sample and should
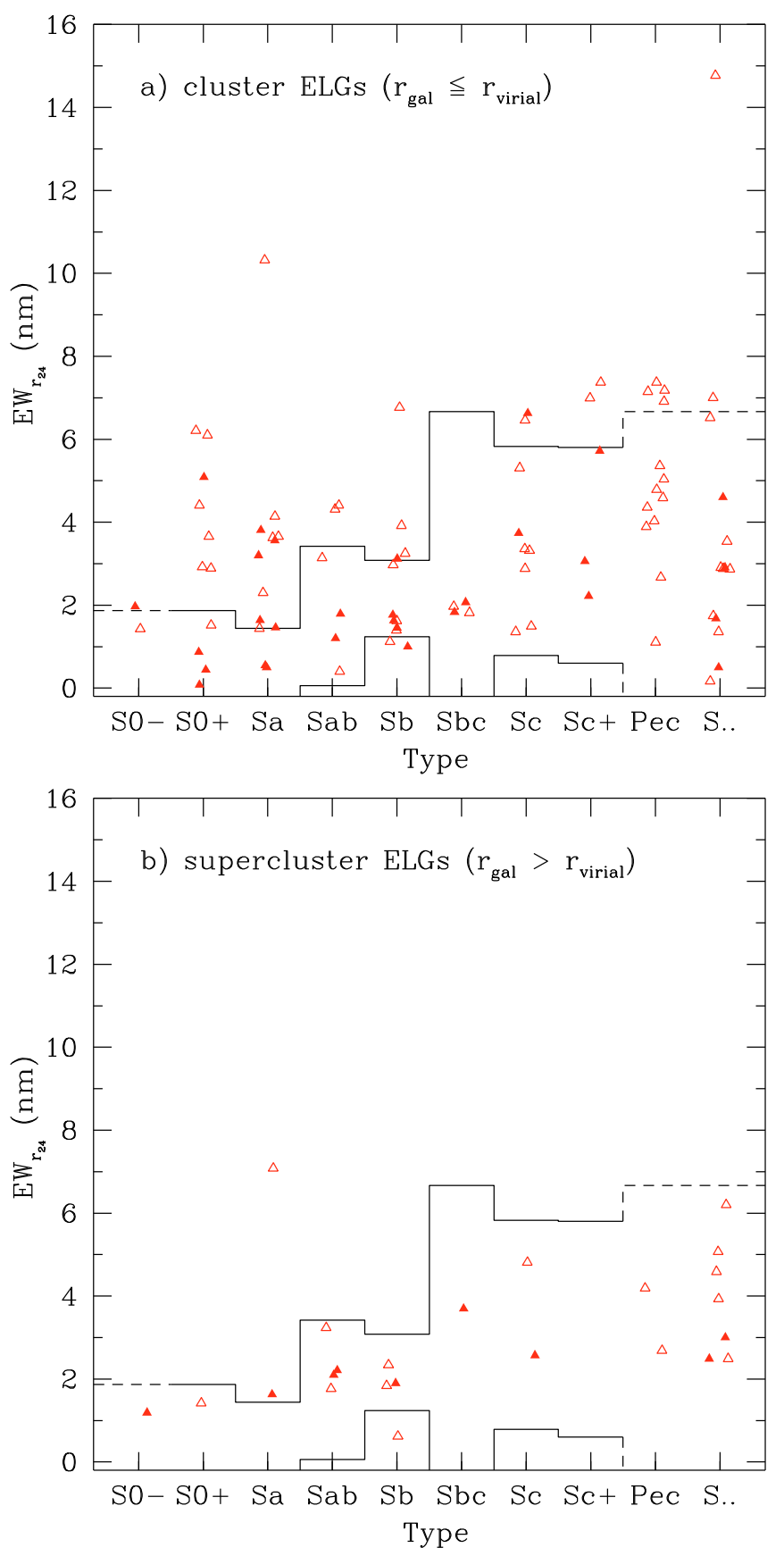

Fig. 3. As Fig. 2 but showing the distribution of $E W$ with Hubble Type for the ELG sample. Dashed lines show assumed limits for ELG types not included in the field sample (see text for details). Typical $E W$ errors are the same as Fig. 2.

therefore provide a conservative limit for these categories, such that any peculiar or unknown spiral galaxies with $E W$ values above this point can also be considered enhanced. For the three E-SO $\left(\mathrm{SO}^{-}\right)$galaxies in the ELG sample it is assumed that the upper field limit is the same as that for the $\mathrm{SO}-\mathrm{SO} / \mathrm{a}\left(\mathrm{SO}^{+}\right)$objects. Again this is likely to be higher than the true value as E-S0 types generally have very little or no current star formation.

Once again, Fig. 3 a shows an increase of $E W$ for a number of cluster galaxies, particularly of types $\mathrm{Sa}-\mathrm{Sb}$, and this enhanced emission also extends to earlier types with seven of $11 \mathrm{~S} 0-\mathrm{S} 0 / \mathrm{a}$ objects (64\%) having $E W$ values enhanced relative to the field. It can also be seen that a few late type Sc and later galaxies as 


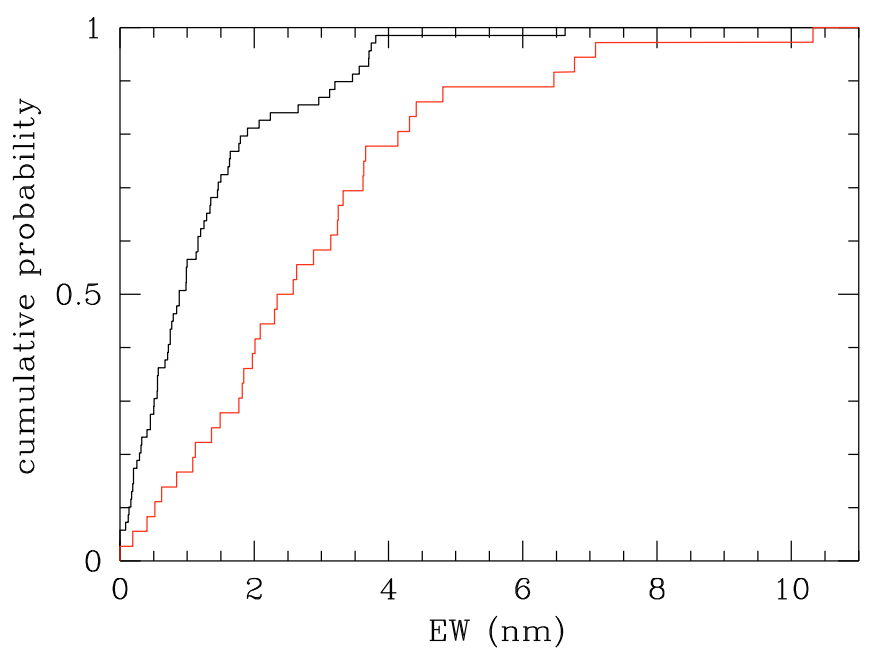

Fig. 4. Cumulative distributions with $E W$ for disturbed (red) and undisturbed (black) galaxies. A K-S test suggests the distributions are significantly different.

well as some peculiar and unclassifiable spiral galaxies appear to have enhanced emission. In contrast, the supercluster galaxies in Fig. $3 \mathrm{~b}$ are almost indistinguishable from the true field sample.

The ELG sample is biased towards objects with more luminous, higher surface brightness $\mathrm{H} \alpha$ emission, and so includes fewer galaxies with low $E W$. The low emission objects seen in the Sa-Sc sample are, therefore, not included in the ELG dataset. However, of the galaxies included in the ELG sample, some 34 objects $(38 \%)$ have enhanced $E W$ values, of which 25 $(\sim 74 \%)$ are classified as disturbed. The non-enhanced ELG objects also have a relatively high proportion of disturbed galaxies, with around $61 \%$ showing signs of tidal disturbance. Even excluding peculiar galaxies, which are, as expected, all disturbed, this figure still stands at $53 \%$, much higher than the $23 \%$ disturbed galaxies seen amongst the $\mathrm{Sa}-\mathrm{Sc}$ non-enhanced objects. This suggests that disturbed galaxies preferentially have brighter $\mathrm{H} \alpha$ emission, even for non-enhanced objects.

Assigning values $0-3$ to the tidal disturbance categories from undisturbed (no rank) to definitely disturbed $(T)$ allows a Kendall rank test to be carried out on the complete Sa-Sc sample. This shows a substantial correlation $(\tau=0.39)$ of $E W$ with tidal disturbance which is significant at the $>5 \sigma$ level. Similarly, a K-S test is performed to compare the distribution of $E W$ values in the disturbed and undisturbed samples. This gives a probability of only $1.1 \times 10^{-5}$ that the data are drawn from the same distribution, showing that the disturbed and undisturbed objects have significantly different $E W$ distributions at $>4 \sigma$ level. The cumulative distributions with $E W$ for the disturbed (red) and undisturbed (black) Sa-Sc galaxies are shown in Fig. 4.

Koopmann \& Kenney (2004b) also detect a number of spirals with star formation rates enhanced by up to a factor of 3 . They find that these are generally lower luminosity galaxies $\left(M_{B}>-18\right)$, for which they lack a good field comparison sample. These authors therefore restrict their sample to galaxies brighter than $M_{R_{24}}=-19.5\left(M_{B} \sim-18.5\right)$ to avoid a possible luminosity bias. Kennicutt et al. (1984) and Boselli et al. (2001) also find that lower luminosity galaxies tend to have higher $E W$ values.

For the full Sa-Sc cluster and supercluster data, a Kendall rank test shows a moderate $(\tau=0.24)$ but significant $(3.7 \sigma)$ dependence of $E W$ on $R$ band magnitude. This result is in agreement with, for example, Gavazzi et al. (1996), who find a

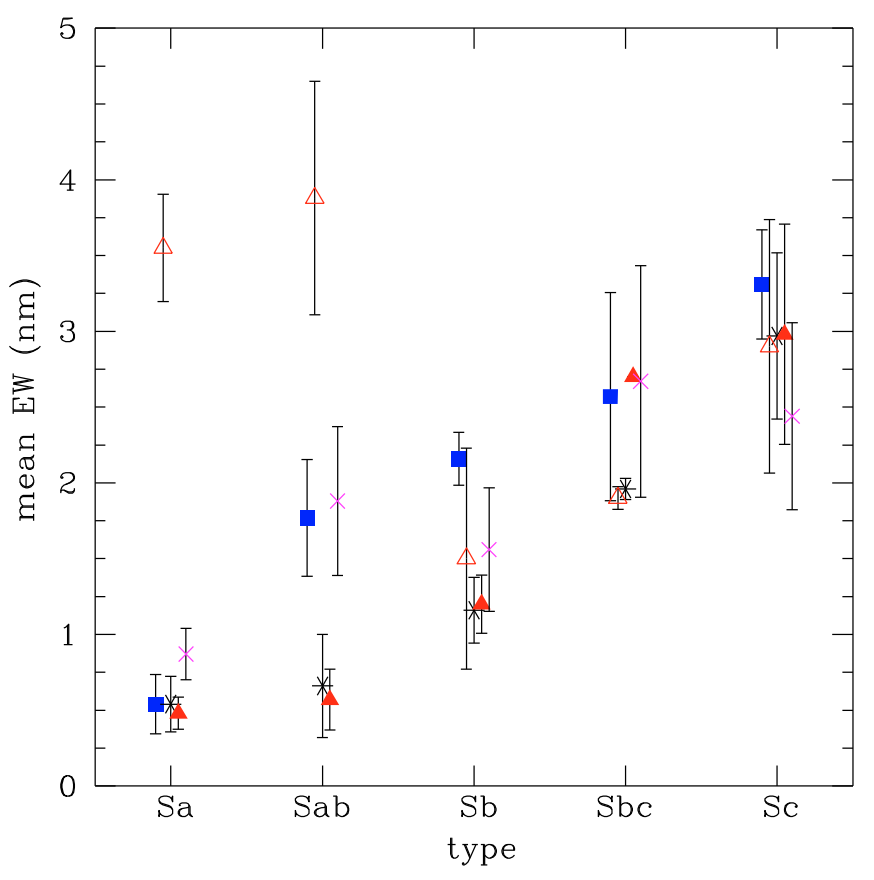

Fig. 5. Mean $E W$ values with type for the $\mathrm{Sa}-\mathrm{Sc}$ cluster sample (stars), the field (squares), and the supercluster field (crosses). The cluster sample is also split into disturbed (open triangles) and undisturbed (closed triangles) galaxies.

significant anti-correlation between galaxy mass and specific star formation rate. The current sample, however, has a limiting magnitude of $M_{B} \sim-18.5$, equivalent to the cut made by Koopmann \& Kenney (2004b), and therefore includes only relatively bright galaxies. The mean magnitude of the cluster sample is also slightly brighter than that of the field galaxies. The correlations found in the present study, and by Gavazzi et al. (1996), between $E W$ and galaxy luminosity would thus predict overall lower $E W$ values for the cluster sample, and cannot be used to explain the enhanced emission of cluster $\mathrm{Sa}-\mathrm{Sc}$ galaxies when compared to the field.

\section{Mean EW values}

Figure 5 shows the mean $E W$ values with type for the $\mathrm{Sa}-\mathrm{Sc}$ cluster (stars), supercluster (crosses) and field (squares) samples. Means are calculated using biweight estimators to reduce the influence of outliers and the error bars shown give the standard error on the mean, $\sigma / \sqrt{n}$. The cluster data are also split into disturbed (open triangles) and undisturbed (closed triangles) galaxies. This split is not done for the supercluster sample due to the small numbers of objects involved (only 31 galaxies in total), however, the mean $E W$ values for the full supercluster sample are generally consistent with the field data.

It can be seen from Fig. 5 that the mean $E W$ values for the full cluster data seem to be reduced in general compared to the field sample, with the exception of Sa galaxies, but this is only significant for $\mathrm{Sab}$ and $\mathrm{Sb}$ types. The undisturbed objects follow the same trend, and with the exception of Sbc types, where only a single object is undisturbed, the undisturbed mean $E W$ values are very similar to those for the full sample. The disturbed objects, on the other hand, show a very different trend, with mean $E W$ values for $\mathrm{Sa}-\mathrm{Sab}$ galaxies greatly enhanced above both total cluster and field values. For later types, however, the mean disturbed $E W$ values are close to, and consistent with, both the 


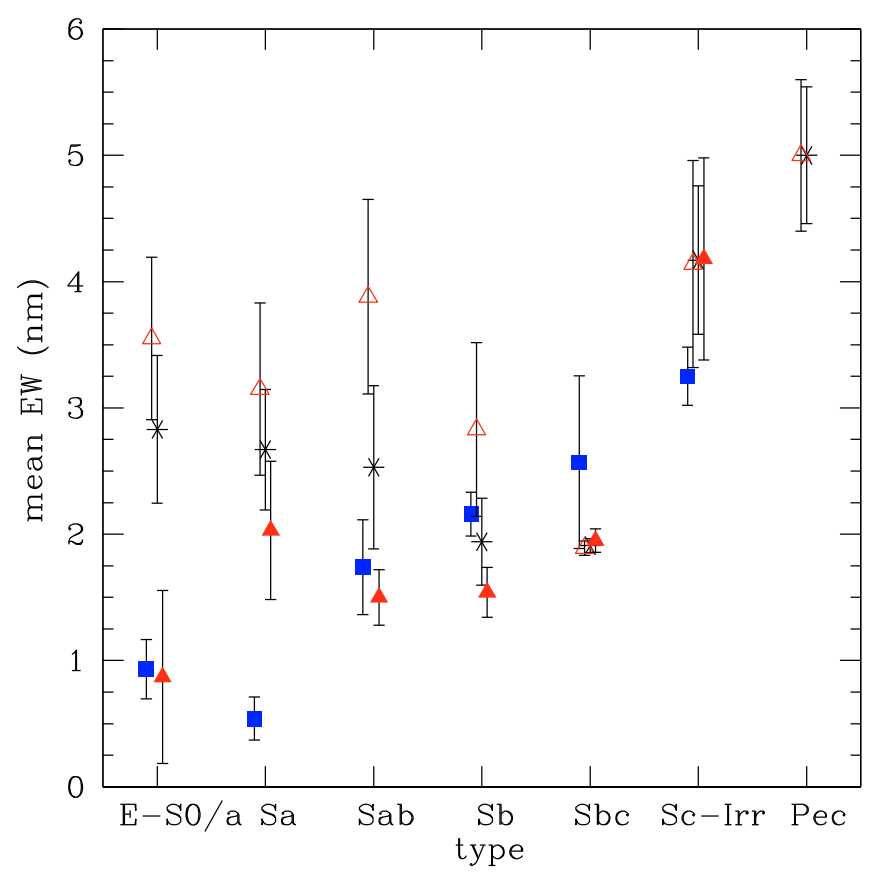

Fig. 6. Mean $E W$ values with type for the cluster ELG sample (stars) split into disturbed (open triangles) and undisturbed (filled triangles) galaxies. The field data are shown as filled squares. Points for the supercluster subset are not shown, because of the small number of galaxies in this category; see Fig. 3.

full and undisturbed cluster samples. Although the highest $E W$ enhancements are seen for the disturbed early type spirals, the fraction of $\mathrm{Sa}-\mathrm{Sab}$ galaxies that are tidally disturbed is only $21 \%$, compared to $48 \%$ and $45 \%$ for $\mathrm{Sb}-\mathrm{Sbc}$ and $\mathrm{Sc}$ galaxies respectively.

A similar plot is shown in Fig. 6 for the cluster ELG sample. Here all E-S0/a cluster galaxies are grouped into a single bin and compared to the field mean S0/a $E W$. In order to increase the numbers of objects in the cluster later type bins, all Sc-Irr galaxies have also been grouped together. Spirals of unclassifiable type are not included in this figure. The undisturbed cluster ELGs have $E W$ values comparable to the field data, however, the ELG sample is biased towards galaxies with brighter $\mathrm{H} \alpha$ emission, so the true mean $E W$ values may be somewhat lower. Once again, the disturbed galaxy sample shows significantly enhanced mean $E W$ values for $\mathrm{Sa}-\mathrm{Sab}$ spirals, but this also extends further to even earlier types (E-S0/a). The sample of peculiar galaxies, all of which are disturbed, has a mean $E W$ greater than any field type. The increases of mean $E W$ in specific Hubble types in Figs. 5 and 6 illustrate that the difference in the overall $E W$ distributions of the disturbed and undisturbed galaxies, shown in Fig. 4, cannot be attributed simply to differences in the morphological makeup of the disturbed and undisturbed samples. Disturbance seems to affect $E W$ in galaxies of a given type, at least for early types.

It is of interest to consider possible reasons for this enhancement in $E W$ being apparent only for early types. Two explanations can be proposed. The first is that enhanced emission tends to take place in the densest cluster regions, where we expect galaxies to be stripped and generally have lower disk emission, thus appearing as early-type galaxies. The second is that the scatter in $E W$ values for late-type unstripped galaxies is much larger than for early types, and may therefore tend to mask any effect.

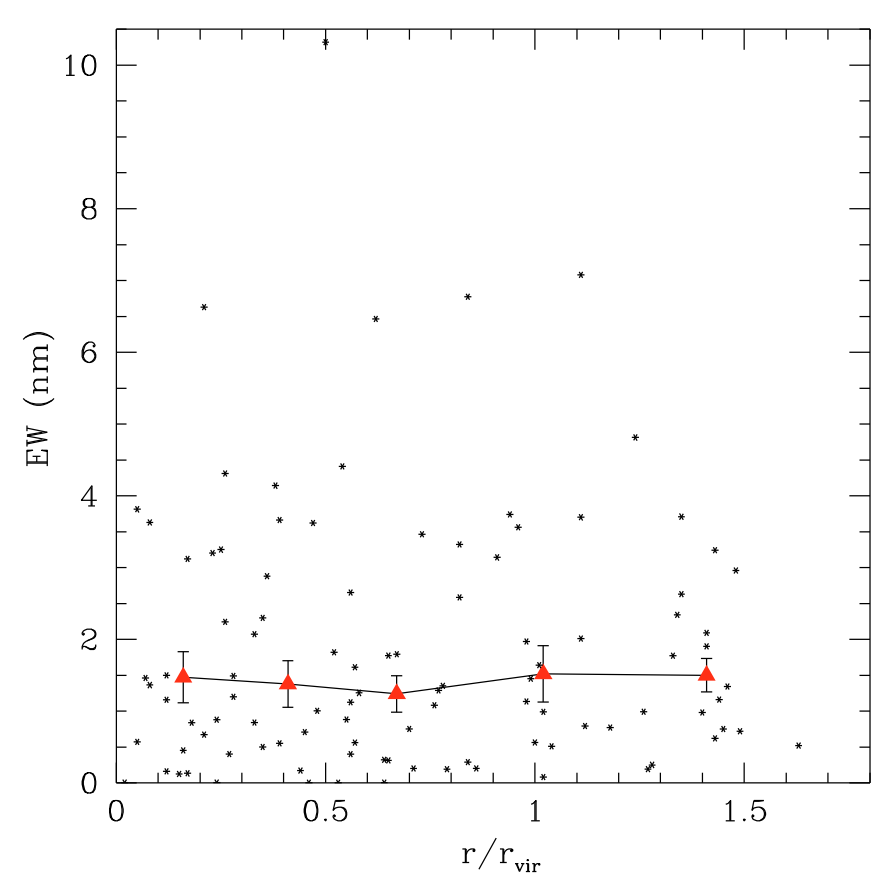

Fig. 7. $\mathrm{H} \alpha E W$ as a function of clustercentric distance for the $\mathrm{Sa}-\mathrm{Sc}$ sample. Large points show mean distances and $E W$ values for bins of equal numbers of galaxies.

\section{Clustercentric radial distribution of $\mathrm{H} \alpha E W$}

Koopmann \& Kenney (2004b) conclude that truncation of the star forming disk, via ICM-ISM stripping, is the dominant process affecting galaxies in clusters. If this is correct then the mean $E W$ of cluster spiral galaxies should decrease towards the cluster centre.

Studies using the 2dF Galaxy Redshift Survey (Lewis et al. 2002) and SDSS (Gómez et al. 2003) have suggested that there is a transition in star formation activity at a characteristic density corresponding to the local density at $\sim 1$ virial radius, although it is difficult to disentangle this from the known morphologydensity relation (Boselli \& Gavazzi 2006). Gavazzi et al. (2006) also find evidence that the average $\mathrm{H} \alpha E W$ of luminous spirals in the Virgo and Coma + Abell 1367 cluster samples decreases in the inner $\sim 1$ virial radius, although the binning of the data results in very few points within $1 r_{\text {vir }}$ (two points for Virgo, one for Coma + A1367) such that it is not possible to trace any gradual variation within the cluster itself. Yuan et al. (2005), however, studied the star formation properties of 184 bright cluster galaxies in the $z \sim 0.08$ cluster A2255 and found that, although there is a slight trend for the specific star formation rates of early-type galaxies to decrease towards the cluster centre, the inner latetype galaxies, in fact, tend to have higher star formation rates.

Figure 7 shows the $E W$ values of the $\mathrm{Sa}-\mathrm{Sc}$ sample plotted against distance in virial radii from the composite cluster centre. Individual galaxies are plotted as black stars, and the red triangles show the mean distance and equivalent width in 5 approximately equal bins, calculated using a biweight estimator. The error bars show the standard error in $E W$ for each bin. The mean $E W$ values in the cluster and supercluster $\mathrm{Sa}-\mathrm{Sc}$ sample are lower than the overall field mean for the Sa-Sc sample, however, this is likely to be due to the different morphological mix in the cluster and field samples. Figure 7, however, shows no change in the star formation rate of late type galaxies with 
C. F. Bretherton et al.: Comparison of integrated properties of cluster and field galaxies

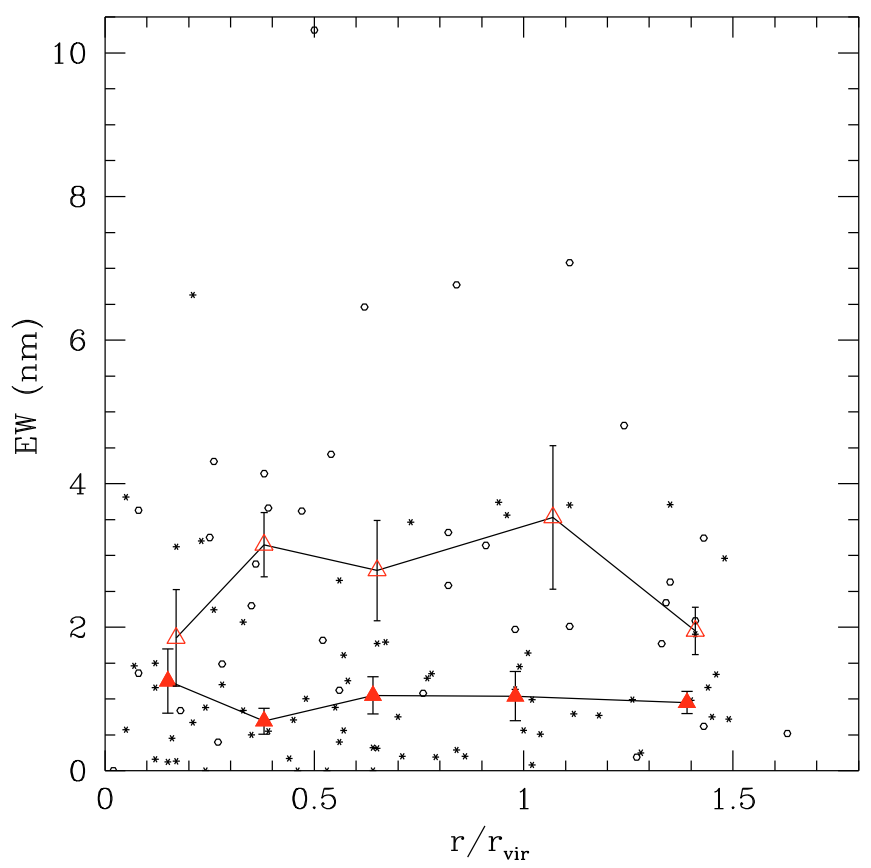

Fig. 8. As Fig. 7, but split into disturbed (open points) and undisturbed (closed points) galaxies.

clustercentric radius. This suggests that stripping alone cannot dominate the transformation of spiral galaxies in these clusters.

It is tempting to suggest that the lack of an observed trend between decreasing star formation and proximity to the cluster centre may be due to field interlopers projected towards the inner parts of the cluster. Moss \& Whittle (2000), however, find that contamination by field galaxies accounts for only $\sim 20 \%$ of spirals within 0.5 Abell radii (which corresponds to roughly $0.5 r_{\text {vir }}$ for the composite cluster), but will be more important outside this radius. Field contamination would therefore likely increase any such trend and cannot account for the flat distribution observed.

Given the observed differences in star formation activity for disturbed and undisturbed galaxies, it is also of interest to study the clustercentric radial distributions of $E W$ values for each sample separately. This is shown in Fig. 8. Here individual undisturbed galaxies are shown as before with black stars, whilst the disturbed population have open points. As with previous plots, the mean values for the undisturbed galaxies are plotted as filled triangles whilst open triangles represent the means for the binned disturbed objects. Figure 8 shows that, as with the complete sample, no change is seen in mean $E W$ with distance from the cluster centre for the undisturbed sample. As expected, the means for the disturbed galaxies are higher than those for the undisturbed objects, but, although the mean star formation appears to be slightly higher at intermediate clustercentric distances, the points and error bars are still consistent with a flat distribution. The same lack of a significant trend is found for the ELG sample, shown in Fig. 9.

This result is initially surprising, given the number of studies finding negative correlations between emission-line strength and the number density of the local environment. This dates back at least as far as Osterbrock (1960), who found emission lines to be less prevalent in elliptical galaxies in dense clusters than in Virgo cluster ellipticals. The same trend was found for larger samples of cluster galaxies, including spiral galaxies, by Gisler (1978) and Dressler et al. (1985). Most recently, Vulcani et al. (2010) conclude that the average SF rate in 604 galaxies within

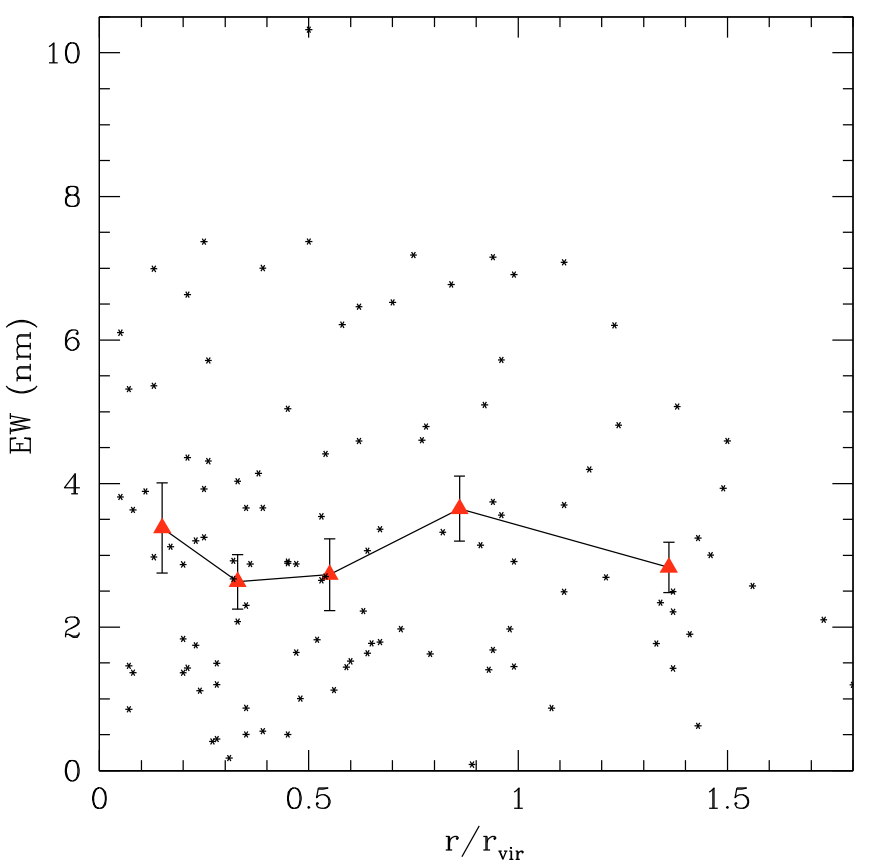

Fig. 9. As Fig. 7, but for the ELG sample.

16 intermediate-redshift clusters varies systematically with environment, even at fixed galaxy mass.

However, other studies have found different results, more in line with those found here. These include Biviano et al. (1997), who found that their overall conclusions regarding the correlation between emission-line strength and environment depended critically on a systematic bias resulting from the different effects of magnitude-limited selection on field and cluster samples. Once this had been corrected for, they found no difference between the emission line properties of field and cluster galaxies of a given morphological type. This result was confirmed by Moss \& Whittle (2005), who further discuss the selection effect analysis of Biviano et al. (1997) and find results similar to those of the present paper for 379 galaxies in low-redshift galaxies, with Objective Prism measures of $\mathrm{H} \alpha$ emission. Finally, Carter et al. (2001) and Rines et al. (2005) both conclude that the distributions of total $\mathrm{H} \alpha E W$ values for galaxies with significant SF show no difference between samples selected within or outside the virial radius of their host clusters.

\section{Distribution of disturbed cluster galaxies}

A comparison of the cumulative distributions of the clustercentric distances of disturbed vs. undisturbed $\mathrm{Sa}-\mathrm{Sc}$ galaxies suggests that the radial distributions of these samples are similar. A K-S test gives a probability of 0.89 that they are drawn from the same parent distribution.

Figure 10, however, shows a rather different conclusion based on the distribution of galaxy velocities within their clusters. The normalised velocity dispersion is shown for all $\mathrm{Sa}-\mathrm{Sc}$ sample galaxies within $1 r_{\text {vir }}$ of the composite cluster centre (open histogram). The sample is also split into disturbed and undisturbed galaxies, and their distributions are plotted separately in the filled red histograms in the centre and top panels respectively. The undisturbed galaxies appear to be fairly centrally peaked, while the disturbed galaxies show a much flatter distribution. The bottom panel in Fig. 10 shows the normalised velocity distribution for the early type elliptical and lenticular 


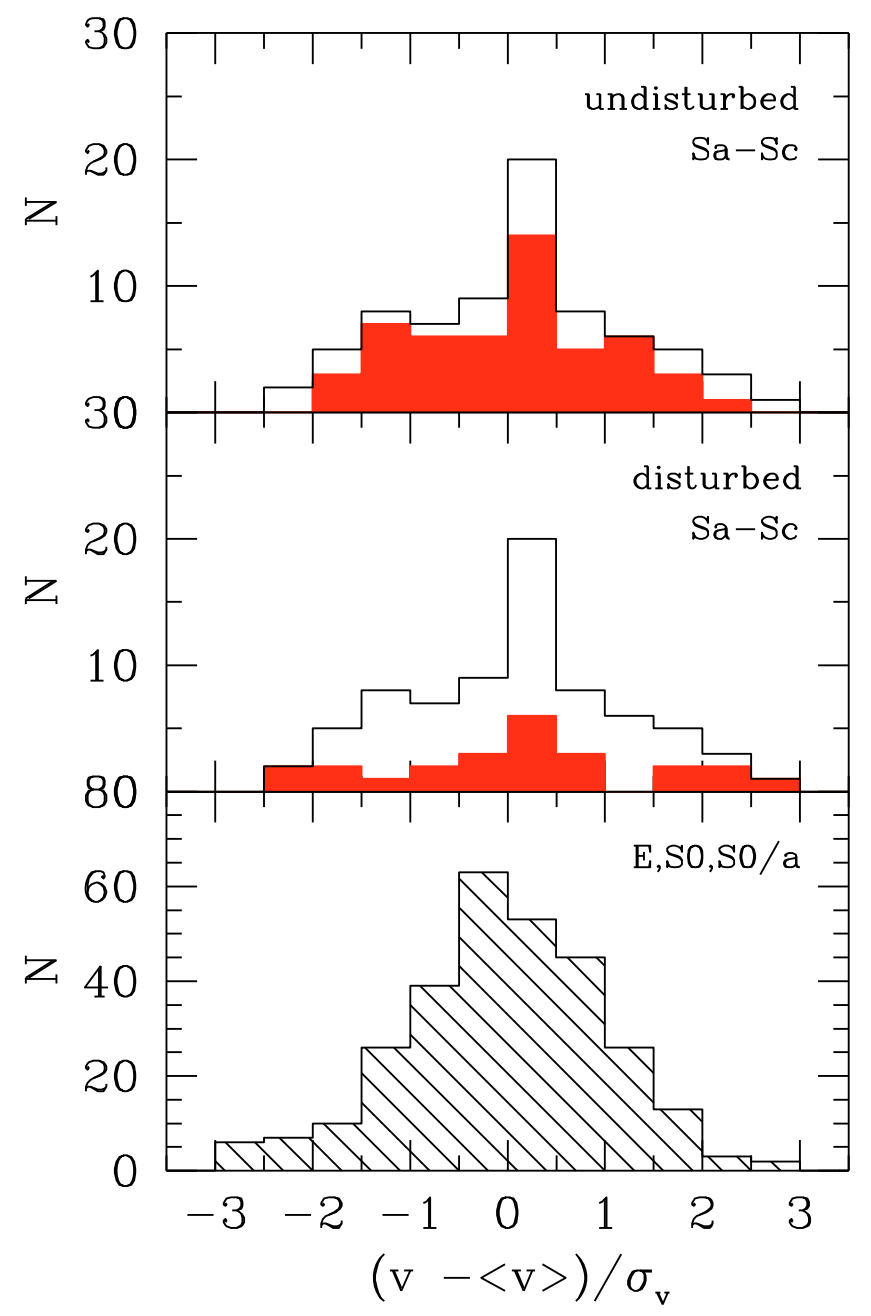

Fig. 10. Normalised velocity distribution for $\mathrm{Sa}-\mathrm{Sc}$ sample galaxies (top and centre), scaled to the cluster mean. Open histograms show the full cluster $\mathrm{Sa}-\mathrm{Sc}$ sample, with filled histograms showing the disturbed (centre) and undisturbed (top) populations. The bottom plot shows the normalised velocity distribution for the early type population in the six clusters included in the $\mathrm{Sa}-\mathrm{Sc}$ sample.

galaxies in the six $\mathrm{Sa}-\mathrm{Sc}$ sample clusters. These objects were used to calculate the cluster means and dispersions that were then employed to normalise the later type population, and they therefore have a mean normalised velocity of 0.0 , with a standard deviation of 1.0.

The mean and dispersion of the velocity distribution have been calculated for each Sa-Sc sample using biweight estimators. The undisturbed sample has a mean of $0.11 \pm 0.14$ with a dispersion of 1.02, and is therefore consistent with the early type population used to calculate the cluster velocity distributions (K-S probability $=0.77$, data folded about $\left.(v-\langle v\rangle) / \sigma_{v}=0\right)$. For the disturbed galaxies, however, although the mean value of $0.09 \pm 0.30$ is still consistent with the composite cluster mean, the dispersion has a much higher value of 1.45. This is, in fact, likely to be a lower limit to the dispersion of disturbed galaxies as the $3 \sigma$ velocity cut applied in the selection of the sample means that galaxies with higher velocity deviations would have been omitted. Even so, a K-S test shows that the velocity distributions of the disturbed $\mathrm{Sa}-\mathrm{Sc}$ sample and the early type population are different at $\gtrsim 2 \sigma$ significance. The distribution of undisturbed galaxies drops off before the $3 \sigma$ limit. Comparing the velocities of the disturbed and undisturbed $\mathrm{Sa}-\mathrm{Sc}$ galaxies, a K-S test gives a probability of 0.06 that these are drawn from the same distribution. The marginally higher velocity dispersion observed for the disturbed galaxies $(\sim \sqrt{2}$ greater than the undisturbed sample) is suggestive of an infalling population, and a similar result was found for the larger sample of cluster galaxies studied by Moss (2006). It should also be noted that the disturbed galaxy Sa-Sc sample studied here has a somewhat later mean type ( $T=2.96$, cf. 2.12 for the undisturbed galaxies) which may have some bearing on the interpretation of this result.

\section{Summary}

Comparison of the global $\mathrm{H} \alpha E W$ values of cluster, supercluster and field galaxies has identified a population of cluster galaxies (particularly early type spirals and lenticulars) with enhanced star formation compared to their field counterparts. These objects are also more likely to have a disturbed appearance than non-enhanced galaxies. Tidal disturbance is found to be correlated with higher $\mathrm{H} \alpha E W$ at $>5 \sigma$ (Kendall rank test). A K-S test also shows that the distributions of $E W$ values in disturbed and undisturbed populations are significantly different $(>4 \sigma)$. This disturbance is seen in the stellar component and hence is indicative of tidal effects, rather than, e.g., ram-pressure stripping. A number of galaxies with unusually weak line emission are also seen in the cluster $\mathrm{Sa}-\mathrm{Sc}$ sample. The supercluster samples, on the other hand, appear very similar to the field.

Comparing the mean $E W$ values for each type suggests that star formation may be reduced in general for undisturbed cluster galaxies across most types. The disturbed galaxies, however, have mean $E W$ values well above those for field early type spirals, but for later types they are consistent with the field and undisturbed cluster samples. The sample of highly disturbed, peculiar galaxies included in the ELG sample has a mean $E W$ higher than any other field or cluster type. These results suggest that galaxy-galaxy interactions and mergers may play a significant role in the evolution of cluster spirals.

A study of the clustercentric radial distribution of $\mathrm{H} \alpha E W$ also shows no correlation between $E W$ and distance from the cluster centre. This suggests that stripping alone, which would lead to a gradual decrease of star formation towards the cluster centre, cannot be solely responsible for the transformation of spiral galaxies in these clusters, and adds further weight to the argument that tidal interactions between galaxies may also be important.

An investigation of the distribution of disturbed and undisturbed galaxies within the cluster shows that, although there appears to be no difference in distribution as a function of clustercentric distance, the disturbed galaxies have a marginally higher velocity dispersion that may indicate an infalling population.

Future papers in this series will look in more detail at the distribution of SF activity within the galaxies studied in the present paper. This analysis will initially use concentration indices as a measure of the compactness of SF, to probe the prevalence of outer truncation and centrally-concentrated starbursts. Radial light profiles will then be used in a more detailed study of these processes.

Acknowledgements. This research has made use of the NASA/IPAC Extragalactic Database (NED) which is operated by the Jet Propulsion Laboratory, California Institute of Technology, under contract with the National Aeronautics and Space Administration. The referee is thanked for many helpful suggestions. C.F.B., P.A.J. and M.W. dedicate this paper to the memory of our respected and sadly-missed colleague, Chris Moss. 


\section{References}

Balick, B., \& Heckman, T. 1981, A\&A, 96, 271

Balogh, M. L., Schade, D., Morris, S. L., et al. 1998, ApJ, 504, L75+

Balogh, M. L., Navarro, J. F., \& Morris, S. L. 2000, ApJ, 540, 113

Barnes, J. E., \& Hernquist, L. 1996, ApJ, 471, 115

Bekki, K. 1999, ApJ, 510, L15

Bekki, K., Couch, W. J., \& Shioya, Y. 2002, ApJ, 577, 651

Bertola, F., \& Sharp, N. A. 1984, MNRAS, 207, 47

Biviano, A., Katgert, P., Mazure, A., et al. 1997, A\&A, 321, 84

Boselli, A., \& Gavazzi, G. 2006, PASP, 118, 517

Boselli, A., Gavazzi, G., Donas, J., \& Scodeggio, M. 2001, AJ, 121, 753

Bower, R. G., \& Balogh, M. L. 2004, in Clusters of Galaxies: Probes of Cosmological Structure and Galaxy Evolution, ed. J. S. Mulchaey, A. Dressler, \& A. Oemler, 325

Butcher, H., \& Oemler, Jr., A. 1978, ApJ, 219, 18

Butcher, H., \& Oemler, Jr., A. 1984, ApJ, 285, 426

Byrd, G., \& Valtonen, M. 1990, ApJ, 350, 89

Carter, B. J., Fabricant, D. G., Geller, M. J., Kurtz, M. J., \& McLean, B. 2001, ApJ, 559, 606

Conselice, C. J., Gallagher, J. S., Calzetti, D., Homeier, N., \& Kinney, A. 2000, AJ, 119, 79

Cowie, L. L., \& Songaila, A. 1977, Nature, 266, 501

de Vaucouleurs, G. 1959, Handbuch der Physik, 53, 275

de Vaucouleurs, G. 1974, in, The Formation and Dynamics of Galaxies, ed. J. R. Shakeshaft, IAU Symp., 58, 1

de Vaucouleurs, G., de Vaucouleurs, A., Corwin, H. G., et al. 1991, Third Reference Catalogue of Bright Galaxies (Berlin Heidelberg New York, Springer-Verlag), 1-3

Donas, J., Milliard, B., Laget, M., \& Buat, V. 1990, A\&A, 235, 60

Dressler, A., Thompson, I. B., \& Shectman, S. A. 1985, ApJ, 288, 481

Dressler, A., Oemler, A. J., Couch, W. J., et al. 1997, ApJ, 490, 577

Finn, R. A., Zaritsky, D., McCarthy, Jr., D. W., et al. 2005, ApJ, 630, 206

Gavazzi, G., \& Contursi, A. 1994, AJ, 108, 24

Gavazzi, G., Pierini, D., \& Boselli, A. 1996, A\&A, 312, 397

Gavazzi, G., Catinella, B., Carrasco, L., Boselli, A., \& Contursi, A. 1998, AJ, 115,1745

Gavazzi, G., Boselli, A., Cortese, L., et al. 2006, A\&A, 446, 839

Gisler, G. R. 1978, MNRAS, 183, 633

Gnedin, O. Y. 2003, ApJ, 582, 141

Gómez, P. L., Nichol, R. C., Miller, C. J., et al. 2003, ApJ, 584, 210

Gunn, J. E., \& Gott, J. R. I. 1972, ApJ, 176, 1

Hashimoto, Y., Oemler, A. J., Lin, H., \& Tucker, D. L. 1998, ApJ, 499, 589

Henriksen, M., \& Byrd, G. 1996, ApJ, 459, 82

Hernquist, L., \& Mihos, J. C. 1995, ApJ, 448, 41
Iono, D., Yun, M. S., \& Mihos, J. C. 2004, ApJ, 616, 199

James, P. A., Shane, N. S., Beckman, J. E., et al. 2004, A\&A, 414, 23

James, P. A., Bretherton, C. F., \& Knapen, J. H. 2009, A\&A, 501, 207

Kennicutt, Jr., R. C., Bothun, G. D., \& Schommer, R. A. 1984, AJ, 89, 1279

Kennicutt, Jr., R. C., Roettiger, K. A., Keel, W. C., van der Hulst, J. M., \& Hummel, E. 1987, AJ, 93, 1011

Kinney, A. L., Bohlin, R. C., Calzetti, D., Panagia, N., \& Wyse, R. F. G. 1993, ApJS, 86, 5

Koopmann, R. A., \& Kenney, J. D. P. 2004a, ApJ, 613, 866

Koopmann, R. A., \& Kenney, J. D. P. 2004b, ApJ, 613, 851

Larson, R. B., Tinsley, B. M., \& Caldwell, C. N. 1980, ApJ, 237, 692

Lavery, R. J., \& Henry, J. P. 1988, ApJ, 330, 596

Lewis, I., Balogh, M., De Propris, R., et al. 2002, MNRAS, 334, 673

Mihos, J. C. 2004, in Clusters of Galaxies: Probes of Cosmological Structure and Galaxy Evolution, ed. J. S. Mulchaey, A. Dressler, \& A. Oemler, 277

Mihos, J. C., Richstone, D. O., \& Bothun, G. D. 1992, ApJ, 400, 153

Moore, B., Lake, G., Quinn, T., \& Stadel, J. 1999, MNRAS, 304, 465

Moran, S. M., Ellis, R. S., Treu, T., et al. 2007, ApJ, 671, 1503

Moss, C. 2006, MNRAS, 373, 167

Moss, C., \& Whittle, M. 1993, ApJ, 407, L17

Moss, C., \& Whittle, M. 2000, MNRAS, 317, 667

Moss, C., \& Whittle, M. 2005, MNRAS, 357, 1337

Moss, C., Whittle, M., \& Pesce, J. E. 1998, MNRAS, 300, 205

Mulder, P. S., \& van Driel, W. 1996, A\&A, 309, 403

Mulder, P. S., van Driel, W., \& Braine, J. 1995, A\&A, 300, 687

Nilson, P. 1973, Uppsala general catalogue of galaxies (Acta Universitatis Upsaliensis. Nova Acta Regiae Societatis Scientiarum Upsaliensis Uppsala Astronomiska Observatoriums Annaler, Uppsala: Astronomiska Observatorium)

Noguchi, M., \& Ishibashi, S. 1986, MNRAS, 219, 305

Nulsen, P. E. J. 1982, MNRAS, 198, 1007

Osterbrock, D. E. 1960, ApJ, 132, 325

Park, C., Choi, Y.-Y., Vogeley, M. S., Gott, J. R. I., \& Blanton, M. R. 2007, ApJ, 658,898

Quilis, V., Moore, B., \& Bower, R. 2000, Science, 288, 1617

Rines, K., Geller, M. J., Kurtz, M. J., \& Diaferio, A. 2005, AJ, 130, 1482

Shane, N. S., \& James, P. A. 2002, in Disks of Galaxies: Kinematics, Dynamics and Peturbations, ed. E. Athanassoula, A. Bosma, \& R. Mujica, ASP Conf. Ser., 275, 323

Spitzer, L. J., \& Baade, W. 1951, ApJ, 113, 413

Thomas, C. F., Moss, C., James, P. A., et al. 2008, A\&A, 486, 755

Valluri, M., \& Jog, C. J. 1990, ApJ, 357, 367

Vulcani, B., Poggianti, B. M., Finn, R. A., et al. 2010, ApJ, 710, L1

Walker, M. F., \& Chincarini, G. 1967, ApJ, 147, 416

Yuan, Q., Zhao, L., Yang, Y., Wen, Z., \& Zhou, X. 2005, AJ, 130, 2559

Zabludoff, A. I., \& Mulchaey, J. S. 1998, ApJ, 498, L5 